\title{
Asymptotic solutions of soliton type of the Korteweg-de Vries equation with variable coefficients and singular perturbation
}

\author{
Samoilenko V. H. ${ }^{1}$, Samoilenko Yu. I. ${ }^{1}$, Limarchenko V. O. ${ }^{1}$, Vovk V. S. ${ }^{1}$, Zaitseva K. S. ${ }^{2}$ \\ ${ }^{1}$ Taras Shevchenko National University of Kyiv, \\ 64 Volodymyrs'ka Str., 01601, Kyiv, Ukraine \\ ${ }^{2}$ Borys Grinchenko Kyiv University, \\ 18/2 Bulvarno-Kudriavska Str., 04053, Kyiv, Ukraine
}

(Received 15 May 2019; Revised 7 November 2019; Accepted 8 November 2019)

\begin{abstract}
The paper deals with the singularly perturbed Korteweg-de Vries equation with variable coefficients. The equation describes wave processes in various inhomogeneous media with variable characteristics and small dispersion. We consider the general algorithm of construction of asymptotic solutions of soliton type to the equation and present its approximate solutions of this type. We analyze properties of the constructed asymptotic solution depending on a small parameter. The results are demonstrated by the examples of the studied equation. We show that for an adequate description of qualitative properties of soliton type solutions to the singularly perturbed $\mathrm{KdV}$ equation with variable coefficients it is necessary to construct at least the first asymptotic approximation, that is, expansion containing both the main and the first term.
\end{abstract}

Keywords: the Korteweg-de Vries equation, singular perturbation, asymptotic solution, solution of soliton type.

2000 MSC: 35Q53, 35B25, 34E20, 34E05

UDC: 517.9

DOI: $10.23939 / \mathrm{mmc} 2019.02 .374$

\section{Introduction}

In mathematical simulation of complex processes non-linear models are often used. Meaningful example of the similar model is the Korteweg-de Vries (KdV) equation [1]

$$
u_{t}+6 u u_{x}+u_{x x x}=0 .
$$

In 1895 this equation was proposed for describing solitary wave discovered by Russell [2]. The KdV equation has become a subject for studying by many researchers in the XX century after description of elastic collision of nonlinear waves that are named as solitons [3]. Later it was found that the same mechanism of collisions is peculiar to many other non-linear models. In particular, soliton waves were found in models of a plasma [3,4], solid-state physics [5], biological systems [6,7], optic systems [8], etc.

The classical KdV equation (1) describes propagation of waves in homogeneous media with nonlinear dispersion. In more general case, when the medium is heterogeneous and its characteristics depend on both time and space variables, we come to considering the Korteweg-de Vries equation with variable coefficients. For example, Maslov V. P., Dobrokhotov Yu. S. and Omelyanov G. A. studied the $\mathrm{KdV}$ type equation of the following form $[9,10]$

$$
u_{t}+\left(\rho_{1}+3 \rho_{2} u\right) u_{x}+\varepsilon^{2} \rho_{3} u_{x x x}+\rho_{4} u=0, \quad x \in \mathbb{R}, \quad t \in[0 ; T],
$$

This research was partially supported by Ministry of Education and Sciences of Ukraine and Taras Shevchenko National University of Kyiv, grant \# 18 BA 038-01. 
where $\rho_{1}=\sqrt{g H(x)}, \rho_{2}=\sqrt{g H^{-1}(x)} / 2, \rho_{3}=\sqrt{g H^{5}(x)} / 6, \rho_{4}=\rho_{1 x} / 2$ and $H(x)>0$ is the depth of a non-perturbed liquid, $g$ is acceleration of the gravity, $\varepsilon$ is a small parameter, which characterizes a value of dispersion. They found approximate (asymptotic) solutions of equation (2) by means of the non-linear WKB method [11].

The constructed asymptotic solutions of equation (2) were called the soliton-like solutions [10], because these solutions are close to soliton solutions by their structure. Thus, the constructed approximate solutions describe a small deformation of soliton solutions of the KdV equation with certain constant coefficients. As a result, the concept of soliton-like solutions of integrable type equations with variable coefficients and small perturbation was proposed.

It should be also mentioned that the non-linear WKB method was proposed in [11] to construct the main term of asymptotic expansion for a quasiperiodic solution of the KdV equation with singular perturbation. Later, the technique turned out to be fruitful for studying various non-linear equations close to integrable ones. In particular, a number of problems were considered for the KdV equation with variable coefficients of the following form [12-14]

$$
\varepsilon^{n} u_{x x x}=a(x, t, \varepsilon) u_{t}+b(x, t, \varepsilon) u u_{x},
$$

where $n$ is a natural number, the functions $a(x, t, \varepsilon), b(x, t, \varepsilon)$ are written as asymptotic series

$$
a(x, t, \varepsilon)=\sum_{j=0}^{\infty} a_{j}(x, t) \varepsilon^{j}, \quad b(x, t, \varepsilon)=\sum_{j=0}^{\infty} b_{j}(x, t) \varepsilon^{j},
$$

the functions $a_{j}(x, t), b_{j}(x, t)$ are infinitely differentiable with respect to variables $(x, t) \in \mathbb{R} \times[0 ; T]$ for all $j \geqslant 0$, and $\varepsilon>0$ is a small parameter.

Namely, the authors found that structure of asymptotic solutions of equation (3) depends essentially on a degree of the small parameter and we developed the algorithms for construction of different kinds of asymptotic soliton-like solutions of equation (3). Such solutions contain both regular and singular parts of the asymptotics, and, moreover, its singular part reflects soliton wave properties. In addition, the main term of the singular part of the asymptotics is a rapidly decreasing function of the phase variable, while in the general case other terms tend to zero when the phase tends to infinity only in the positive direction and do not have similar properties in the negative direction.

In this paper, we study asymptotic soliton-like solutions of equation (3) for $n=2$. We consider the case when the singular part of the asymptotics tends to zero as its phase tends to infinity in both positive and negative directions. In other words, these functions belong to the space of rapidly decreasing functions of the phase variable. These asymptotic expansions for the KdV equation (3) are called as asymptotic soliton type solutions. It is clear that asymptotic soliton type solutions is a particular case of asymptotic soliton-like solutions which has the important physical sense.

Our main purpose is to perform comparative analysis of the asymptotic soliton type solutions with the corresponding exact solutions of the KdV equation (3). To this purpose we initially briefly describe the algorithm of the constructing asymptotic one-phase soliton-like solutions of equation (3) and analyze its basic stages. We select the asymptotic one-phase solutions of soliton type among these approximate solutions. Further we consider in details the properties of the asymptotic solutions when small parameter $\varepsilon$ tends to zero. For this we consider the asymptotic solutions of soliton type of equation (3) with certain variable coefficients as the example. We show that for an adequate description of qualitative properties of soliton type solutions of the singularly perturbed KdV equation with variable coefficients it is necessary to construct at least the first asymptotic approximation, i.e., the expansion containing both the main and the first terms. 


\section{Preliminary notes and notations}

Asymptotic one-phase soliton-like solution of equation (3) can be written as [12]

$$
u(x, t, \varepsilon)=U_{N}(x, t, \varepsilon)+V_{N}(x, t, \tau, \varepsilon)+O\left(\varepsilon^{N+1}\right),
$$

where

$$
U_{N}(x, t, \varepsilon)=\sum_{j=0}^{N} \varepsilon^{j} u_{j}(x, t)
$$

is the regular part of the asymptotics and the functions $u_{j}(x, t), j=\overline{0, N}$, are infinitely differentiable, and

$$
V_{N}(x, t, \tau, \varepsilon)=\sum_{j=0}^{N} \varepsilon^{j} V_{j}(x, t, \tau), \quad \tau=\frac{x-\varphi(t)}{\varepsilon},
$$

is the singular part of the asymptotics.

Here $\varphi(t) \in C^{\infty}([0 ; T])$ is a scalar function which defines the so-called discontinuity curve $[9,10]$ : $\Gamma=\{(x, t) \in \mathbb{R} \times[0 ; T]: x=\varphi(t)\}$. The value $x-\varphi(t)$ in (7) is called a phase of the one-phase soliton-like function (5).

It should be noted that the regular part of the asymptotics is the background function and the singular part of the asymptotics reflects soliton properties of the desired approximate solution. The latter is taken into account on defining the functional spaces, to which terms of the singular part (5) should belong. Therefore, the functions $V_{0}(x, t, \tau) \in G_{1}^{0}, V_{j}(x, t, \tau) \in G_{1}, j=\overline{1, N}$, where $G_{1}^{0}, G_{1}$ are the functional spaces described below.

The spaces $G_{1}^{0}, G_{1}$ are defined as follows [10]. $G_{1}=G_{1}(\mathbb{R} \times[0 ; T] \times \mathbb{R})$ is a linear space of infinitely differentiable functions $f=f(x, t, \tau),(x, t, \tau) \in \mathbb{R} \times[0 ; T] \times \mathbb{R}$, such that for any non-negative integers $n, p, q, r$, uniformly with respect to $(x, t)$ on every compact set $K \subset \mathbb{R} \times[0 ; T]$ the following conditions hold

$1^{\circ}$. the relation

$$
\lim _{\tau \rightarrow+\infty} \tau^{n} \frac{\partial^{p}}{\partial x^{p}} \frac{\partial^{q}}{\partial t^{q}} \frac{\partial^{r}}{\partial \tau^{r}} f(x, t, \tau)=0, \quad(x, t) \in K,
$$

takes place;

$2^{\circ}$. there exists an infinitely differentiable function $f^{-}(x, t)$ such that

$$
\lim _{\tau \rightarrow-\infty} \tau^{n} \frac{\partial^{p}}{\partial x^{p}} \frac{\partial^{q}}{\partial t^{q}} \frac{\partial^{r}}{\partial \tau^{r}}\left(f(x, t, \tau)-f^{-}(x, t)\right)=0, \quad(x, t) \in K .
$$

The space $G_{1}^{0}=G_{1}^{0}(\mathbb{R} \times[0 ; T] \times \mathbb{R})$ is a space of functions $f=f(x, t, \tau)$ from the space $G_{1}$ such that the following condition

$$
\lim _{\tau \rightarrow-\infty} f(x, t, \tau)=0
$$

holds uniformly with respect to variables $(x, t)$ on every compact $K \subset \mathbb{R} \times[0 ; T]$.

Remark 1. A function from the space $G_{1}^{0}$ is a rapidly decreasing with phase variable $\tau$ function, while a function from the space $G_{1}$ is a step-like function, i.e. it is a rapidly decreasing function when the phase variable $\tau \rightarrow+\infty$ and it has non-zero limit when $\tau \rightarrow-\infty$.

\section{Scheme of construction of the asymptotic solution}

Initially, we look for the asymptotic one-phase soliton-like solutions of equation (3) as (5), and later we select the asymptotic one-phase soliton type solutions among them. It should be noted that the regular part of the asymptotics is the same. So, in contrast to paper [12] we have to find the singular part of 
the asymptotics, all terms of which belong to the space $G_{1}^{0}$. Since the coefficients of equation (3) have general form, it is clear that the problem has no solution for arbitrary functions $a(x, t, \varepsilon), b(x, t, \varepsilon)$. It means that some additional conditions for coefficients of equation (3) must hold.

\subsection{Determination of terms of the regular part}

According to the non-linear WKB technique $[9,11,12]$, for the functions $u_{j}(x, t), j=\overline{0, N}$, we have the following system of partial differential equations

$$
\begin{aligned}
& a_{0}(x, t) \frac{\partial u_{0}}{\partial t}+b_{0}(x, t) \frac{\partial u_{0}}{\partial x} u_{0}=0 \\
& a_{0}(x, t) \frac{\partial u_{j}}{\partial t}+b_{0}(x, t) u_{0}(x, t) \frac{\partial u_{j}}{\partial x}+b_{0}(x, t) u_{j}(x, t) \frac{\partial u_{0}}{\partial x}=f_{j}\left(x, t, u_{0}, u_{1}, \ldots, u_{j-1}\right), \quad j=\overline{1, N}
\end{aligned}
$$

where $f_{j}\left(x, t, u_{0}, u_{1}, \ldots, u_{j-1}\right), j=\overline{1, N}$, are calculated recurrently. In particular, we have

$$
\begin{aligned}
f_{1}\left(x, t, u_{0}\right) & =-a_{1}(x, t) \frac{\partial u_{0}}{\partial t}-b_{1}(x, t) u_{0} \frac{\partial u_{0}}{\partial x}, \\
f_{2}\left(x, t, u_{0}, u_{1}\right) & =-a_{2}(x, t) \frac{\partial u_{0}}{\partial t}-b_{2}(x, t) u_{0} \frac{\partial u_{0}}{\partial x}-a_{1}(x, t) \frac{\partial u_{1}}{\partial t}-b_{1}(x, t) \frac{\partial}{\partial x}\left(u_{0} u_{1}\right)-b_{0}(x, t) u_{1} \frac{\partial u_{1}}{\partial x} .
\end{aligned}
$$

Quasi-linear equation (8) is the Hopf equation with variable coefficients [15], while equation (9) is linear. Their solutions can be easily found in general form by means of the method of characteristics [16]. So, the terms of the regular part of asymptotic (5) are supposed to be known.

\subsection{Determination of terms of the singular part of the asymptotic soliton-like solutions}

The terms of the singular part of asymptotic (5) satisfy the following system of partial differential equations

$$
\begin{aligned}
& \frac{\partial^{3} V_{0}}{\partial \tau^{3}}+a_{0}(x, t) \frac{\partial V_{0}}{\partial \tau} \varphi^{\prime}(t)-b_{0}(x, t)\left(u_{0} \frac{\partial V_{0}}{\partial \tau}+V_{0} \frac{\partial V_{0}}{\partial \tau}\right)=0 \\
& \frac{\partial^{3} V_{j}}{\partial \tau^{3}}+a_{0}(x, t) \frac{\partial V_{j}}{\partial \tau} \varphi^{\prime}(t)-b_{0}(x, t)\left(u_{0} \frac{\partial V_{j}}{\partial \tau}+\frac{\partial}{\partial \tau}\left(V_{0} V_{j}\right)\right)=F_{j}(x, t, \tau),
\end{aligned}
$$

where $F_{j}(x, t, \tau)=F_{j}\left(t, V_{0}(x, t, \tau), \ldots, V_{j-1}(x, t, \tau), u_{0}(x, t), \ldots, u_{j}(x, t)\right)$, are recurrently found by the functions $u_{0}(x, t), u_{1}(x, t), \ldots, u_{j}(x, t), V_{0}(x, t, \tau), V_{1}(x, t, \tau), \ldots, V_{j-1}(x, t, \tau), j=\overline{1, N}$.

The singular terms should satisfy the equations (10), (11) as well as to have certain properties that are peculiar to soliton solutions. In other words, these functions must belong to the space $G_{1}^{0}$. It is also necessary to find the function $\varphi=\varphi(t)$ defined the discontinuity curve $\Gamma$.

The problem is solved in the following special way.

1. Firstly, the function $\varphi(t)$ is supposed to be apriori known and equations (10), (11) are studied on the discontinuity curve $\Gamma$.

2. On studying the function $V_{0}(x, t, \tau), \ldots, V_{j-1}(x, t, \tau), j=\overline{1, N}$, on the curve $\Gamma$ we get the second order nonlinear ordinary differential equation for the function $\varphi=\varphi(t)$. So, further the function $\varphi(t)$ is supposed to be known on an interval $[0 ; T]$.

3. In the next stage, the functions $V_{0}(x, t, \tau), \ldots, V_{j-1}(x, t, \tau), j=\overline{1, N}$, are constructed in such a way that the terms of the singular part (5) of the obtained solution belong to the space $G_{1}^{0}$.

Let us consider these stages in details. 


\subsubsection{Singular terms on the discontinuity curve}

We use the notation $v_{j}=v_{j}(t, \tau)=\left.V_{j}(x, t, \tau)\right|_{x=\varphi(t)}, j=\overline{0, N}$. The functions $v_{j}=v_{j}(t, \tau), j=\overline{1, N}$, satisfy partial differential equations

$$
\begin{aligned}
& \frac{\partial^{3} v_{0}}{\partial \tau^{3}}+a_{0}(\varphi, t) \frac{\partial v_{0}}{\partial \tau} \varphi^{\prime}(t)-b_{0}(\varphi, t)\left[u_{0}(\varphi, t) \frac{\partial v_{0}}{\partial \tau}+v_{0} \frac{\partial v_{0}}{\partial \tau}\right]=0 \\
& \frac{\partial^{3} v_{j}}{\partial \tau^{3}}+a_{0}(\varphi, t) \frac{\partial v_{j}}{\partial \tau} \varphi^{\prime}(t)-b_{0}(\varphi, t)\left[u_{0}(\varphi, t) \frac{\partial v_{j}}{\partial \tau}+v_{j} \frac{\partial v_{0}}{\partial \tau}+v_{0} \frac{\partial v_{j}}{\partial \tau}\right]=\mathcal{F}_{j}(t, \tau)
\end{aligned}
$$

where $\mathcal{F}_{j}(t, \tau)=\left.F_{j}\left(t, V_{0}(x, t, \tau), \ldots, V_{j-1}(x, t, \tau), u_{0}(x, t), \ldots, u_{j}(x, t)\right)\right|_{x=\varphi(t)}$, are found recurrently after determining the functions $u_{0}(x, t), u_{1}(x, t), \ldots, u_{j}(x, t), V_{0}(x, t, \tau), V_{1}(x, t, \tau), \ldots, V_{j-1}(x, t, \tau)$, $j=\overline{1, N}$. In particular,

$$
\begin{gathered}
\mathcal{F}_{1}(t, \tau)=a_{0}(\varphi, t) \frac{\partial v_{0}}{\partial t}+b_{0}(\varphi, t) u_{0 x}(\varphi, t) v_{0}-\left[a_{1}(\varphi, t) \varphi^{\prime}(t)-b_{1}(\varphi, t) u_{0}(\varphi(t), t)-b_{0}(\varphi, t) u_{1}(\varphi, t)\right] \frac{\partial v_{0}}{\partial \tau} \\
-\tau\left[a_{0 x}(\varphi, t) \varphi^{\prime}(t)-b_{0 x}(\varphi, t) u_{0}(\varphi, t)-b_{0}(\varphi, t) u_{0 x}(\varphi, t)\right] \frac{\partial v_{0}}{\partial \tau}+b_{1}(\varphi, t) \frac{\partial v_{0}}{\partial \tau} v_{0}+\tau b_{0 x}(\varphi, t) \frac{\partial v_{0}}{\partial \tau} v_{0} .
\end{gathered}
$$

Let us pass to the analysis of equations (12), (13). Solutions of equation (12) can be found explicitly. The solution in the space $G_{1}^{0}$ can be written as follows

$$
v_{0}(t, \tau)=-3 \frac{A\left(\varphi, \varphi^{\prime}, t\right)}{b_{0}(\varphi, t)} \cosh ^{-2}\left(\sqrt{A\left(\varphi, \varphi^{\prime}, t\right)} \frac{\tau+c_{0}(t)}{2}\right),
$$

where

$$
A\left(\varphi, \varphi^{\prime}, t\right)=-a_{0}(\varphi, t) \varphi^{\prime}(t)+b_{0}(\varphi, t) u_{0}(\varphi, t), \quad \varphi=\varphi(t) .
$$

It is supposed here that $A\left(\varphi, \varphi^{\prime}, t\right)>0$ for all $t \in[0 ; T]$, where $c_{0}(t)$ is an integration constant.

Let us consider now equation (13) for the functions $v_{j}(t, \tau), j=\overline{1, N}$. According to constructing $\mathcal{F}_{1}(t, \tau) \in G_{1}^{0}$, while the function $\mathcal{F}_{j}(t, \tau)$ belongs only to the space $G_{1}$ for every $j=\overline{2, N}$.

The solution of equation (13) belongs to the space $G_{1}$ due to property $\mathcal{F}_{j}(t, \tau) \in G_{1}^{0}, j=\overline{1, N}$, and the orthogonality condition [12]

$$
\int_{-\infty}^{+\infty} \mathcal{F}_{j}(t, \tau) v_{0}(t, \tau) d \tau=0, \quad j=\overline{1, N}
$$

These assumptions are supposed further to be fulfilled.

\subsubsection{Determination of the phase function}

From condition (16) for $j=1$ we get the differential equation for the function $\varphi(t)$ as

$$
\begin{array}{r}
15 a_{0}(\varphi, t) b_{0}(\varphi, t) \frac{d}{d t} A\left(\varphi, \varphi^{\prime}, t\right)+\left[\left(10 a_{0 x}(\varphi, t) b_{0}(\varphi, t)-36 a_{0}(\varphi, t) b_{0 x}(\varphi, t)\right) \varphi^{\prime}+10 b_{0}^{2}(\varphi, t) u_{0 x}(\varphi, t)\right. \\
\left.+3\left(b_{0}^{2}(\varphi, t)\right)_{x} u_{0}(\varphi, t)-20 a_{0}(\varphi, t) b_{0 t}(\varphi, t)\right] A\left(\varphi, \varphi^{\prime}, t\right)=0 .
\end{array}
$$

The relation (17) is the non-linear ordinary differential equation with variable coefficients defined only by the functions $a_{0}(x, t), b_{0}(x, t), u_{0}(x, t)$. It is assumed further that differential equation (17) has a solution. This is possible under rather general conditions for the functions $a_{0}(x, t), b_{0}(x, t)$. So, we can suppose that equation (17) has a solution on certain finite time interval $[0 ; T]$. Note, that in the case of the classical KdV equation with singular perturbation (1) corresponding the equation for finding the function $\varphi=\varphi(t)$ is simple $\frac{d \varphi}{d t}=a^{2}$, where $a$ is an arbitrary non-zero constant. 


\subsubsection{Representation of the singular terms on the discontinuity curve}

Under orthogonality condition (16), solution of equation (13) can be written as [9]

$$
v_{j}(t, \tau)=\nu_{j}(t) \eta_{j}(t, \tau)+\psi_{j}(t, \tau), \quad j=\overline{1, N},
$$

where the function $\eta_{j}(t, \tau) \in G_{1}$ and $\lim _{\tau \rightarrow-\infty} \eta_{j}(t, \tau)=1 ; \psi_{j}(t, \tau) \in G_{1}^{0}$.

Here, we denote

$$
\begin{gathered}
\nu_{j}(t)=\left[a_{0}(\varphi(t), t) \varphi^{\prime}(t)-b_{0}(\varphi(t), t) u_{0}(\varphi(t), t)\right]^{-1} \lim _{\tau \rightarrow-\infty} \Phi_{j}(t, \tau), \\
\Phi_{j}(t, \tau)=\int_{-\infty}^{\tau} \mathcal{F}_{j}(t, \xi) d \xi+E_{j}(t),
\end{gathered}
$$

where the integration constant $E_{j}(t)$ satisfies the following condition

$$
\lim _{\tau \rightarrow+\infty} \Phi_{j}(t, \tau)=0
$$

In particular, we have

$$
\begin{aligned}
& \Phi_{1}(t, \tau)= 6\left[a_{0}(\varphi, t) \frac{d}{d t}\left(\frac{\sqrt{A\left(\varphi, \varphi^{\prime}, t\right)}}{b_{0}(\varphi, t)}\right)+\sqrt{A\left(\varphi, \varphi^{\prime}, t\right)} u_{0 x}+\frac{A\left(\varphi, \varphi^{\prime}, t\right) \sqrt{A\left(\varphi, \varphi^{\prime}, t\right)}}{b_{0}^{2}(\varphi, t)} b_{0 x}(\varphi, t)\right. \\
&+\left.\frac{\sqrt{A\left(\varphi, \varphi^{\prime}, t\right)}}{b_{0}(\varphi, t)}\left[a_{0 x}(\varphi, t) \varphi^{\prime}-\left(b_{0}(\varphi, t) u_{0}(\varphi, t)\right)_{x}\right]\right]\left(1-\tanh \left(\sqrt{A\left(\varphi, \varphi^{\prime}, t\right)} \frac{\tau+c_{0}(t)}{2}\right)\right) \\
&-3\left[\frac{a_{0}(\varphi, t)}{b_{0}(\varphi, t)} \frac{\tau+c_{0}(t)}{2} \frac{d}{d t} A\left(\varphi, \varphi^{\prime}, t\right)-\frac{A\left(\varphi, \varphi^{\prime}, t\right)}{b_{0}(\varphi, t)}\left[a_{1}(\varphi, t) \varphi^{\prime}-\left(b_{0}(\varphi, t) u_{0}(\varphi, t)\right)_{x}\right]\right. \\
&\left.+\frac{A\left(\varphi, \varphi^{\prime}, t\right)}{b_{0}(\varphi, t)} a_{0}(\varphi, t) c_{0}^{\prime}(t)-\tau \frac{A\left(\varphi, \varphi^{\prime}, t\right)}{b_{0}(\varphi, t)}\left[a_{0 x}(\varphi, t) \varphi^{\prime}-\left(b_{0}(\varphi, t) u_{0}(\varphi, t)\right)_{x}\right]\right] \\
& \quad \times \cosh ^{-2}\left(\sqrt{A\left(\varphi, \varphi^{\prime}, t\right)} \frac{\tau+c_{0}(t)}{2}\right)+\frac{9}{2} \frac{A^{2}\left(\varphi, \varphi^{\prime}, t\right)}{b_{0}^{2}(\varphi, t)}\left[b_{0 x}(\varphi, t) \tau+b_{1}(\varphi, t)\right] \\
& \quad \times \cosh ^{-4}\left(\sqrt{A\left(\varphi, \varphi^{\prime}, t\right)} \frac{\tau+c_{0}(t)}{2}\right)-3 \frac{A\left(\varphi, \varphi^{\prime}, t\right) \sqrt{A\left(\varphi, \varphi^{\prime}, t\right)}}{b_{0}^{2}(\varphi, t)} b_{0 x}(\varphi, t) \\
& \quad \times \tanh \left(\sqrt{A\left(\varphi, \varphi^{\prime}, t\right)} \frac{\tau+c_{0}(t)}{2}\right) \cosh ^{-2}\left(\sqrt{A\left(\varphi, \varphi^{\prime}, t\right)} \frac{\tau+c_{0}(t)}{2}\right),
\end{aligned}
$$

where $\varphi=\varphi(t)$.

\subsection{Determination of terms of the singular part of the asymptotic soliton type solutions}

It should be noted that the function $v_{j}(t, \tau), j=\overline{1, N}$, belongs to the space $G_{1}^{0}$ if and only if the condition

$$
\lim _{\tau \rightarrow-\infty} \Phi_{j}(t, \tau)=0, \quad j=\overline{1, N}
$$

is satisfied. Thus, the functions $v_{j}(t, \tau), j=\overline{0, N}$ can be taken as terms of the singular part of asymptotics (5) $V_{j}(x, t, \tau), j=\overline{0, N}$. So, the terms of the singular part of asymptotic (5) are also defined and they are functions of soliton type of the phase variable.

Remark 2. From formula (20) and condition (21) we conclude that the coefficients $a_{0}(\varphi, t), b_{0}(\varphi, t)$ as well as the main term of the regular part $u_{0}(\varphi, t)$ and the phase function $\varphi(t)$ satisfy the following relation 


$$
\begin{array}{r}
a_{0}(\varphi, t) \frac{d}{d t}\left(\frac{\sqrt{A\left(\varphi, \varphi^{\prime}, t\right)}}{b_{0}(\varphi, t)}\right)+\sqrt{A\left(\varphi, \varphi^{\prime}, t\right)} u_{0 x}+\frac{\sqrt{A\left(\varphi, \varphi^{\prime}, t\right)}}{b_{0}(\varphi, t)}\left[a_{0 x}(\varphi, t) \varphi^{\prime}-\left(b_{0}(\varphi, t) u_{0}(\varphi, t)\right)_{x}\right] \\
+\frac{A\left(\varphi, \varphi^{\prime}, t\right) \sqrt{A\left(\varphi, \varphi^{\prime}, t\right)}}{b_{0}^{2}(\varphi, t)} b_{0 x}(\varphi, t)=0, \quad t \in[0 ; T] .
\end{array}
$$

Formula (22) must be taken into account on construction of asymptotic soliton type solutions of the $\mathrm{KdV}$ equation (3) with certainly selected coefficients in the form of a condition for coefficients $a_{0}(x, t)$, $b_{0}(x, t)$, the main term of the regular part of asymptotics $u_{0}(x, t)$ and the phase function $\varphi(t)$.

\section{Small deformations of the soliton type solutions}

Let us show the obtained results for the KdV equation with variable coefficients and singular perturbation when its coefficients match condition (22). We consider the case of the zero background, i.e. when $U_{N}(x, t) \equiv 0$. In this case, the asymptotic one-phase soliton type solutions contain only the singular part describing soliton properties of the solutions.

\subsection{The case of constant coefficients}

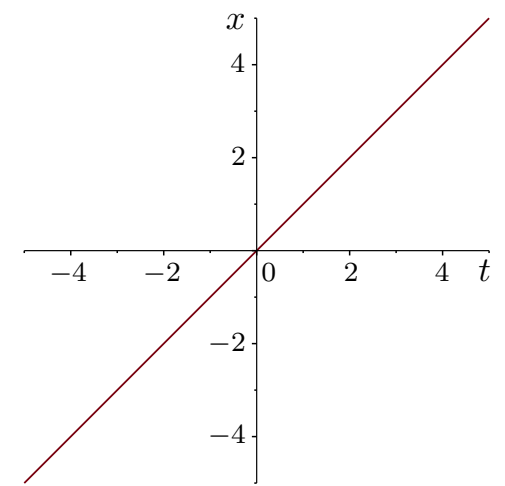

Fig. 1. The discontinuity curve $x-a^{2} t=0$ as $a=1$.

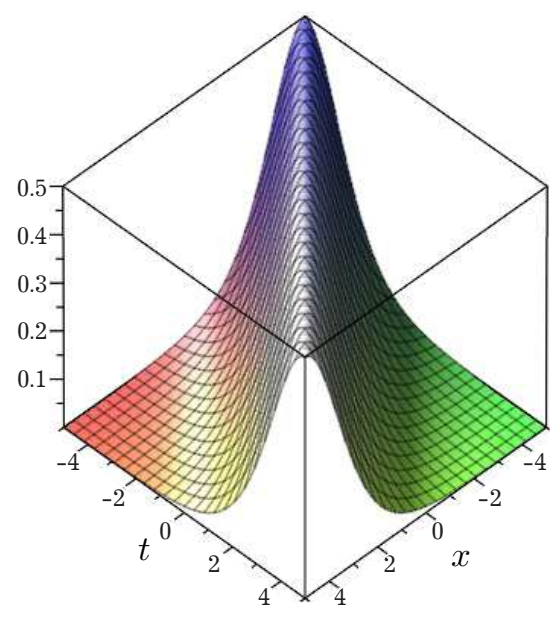

Fig. 2. Solution (23) as $a=1$ and $\varepsilon=1$.
In the case of constant coefficients, by means of the calibre transformation the equation (3) is reduced to the classical $\mathrm{KdV}$ equation with singular perturbation (1). Asymptotic solution of equation (1), constructed by means of the nonlinear WKB method, contains only the main term of the singular part of asymptotics (5) in the following form

$$
u(x, t, \varepsilon)=\frac{a^{2}}{2} \cosh ^{-2}\left(\frac{a \tau}{2}\right), \quad \tau=\frac{x-a^{2} t}{\varepsilon},
$$

where $a>0$ is an arbitrary constant.

Moreover, formula (23) represents the exact solution of equation (1).

Here, the phase is given by the function $\varphi(t)=a^{2} t$, defined for all values of $t$. The discontinuity curve is given by the equation $x-a^{2} t=0$ (see Fig. 1 ).

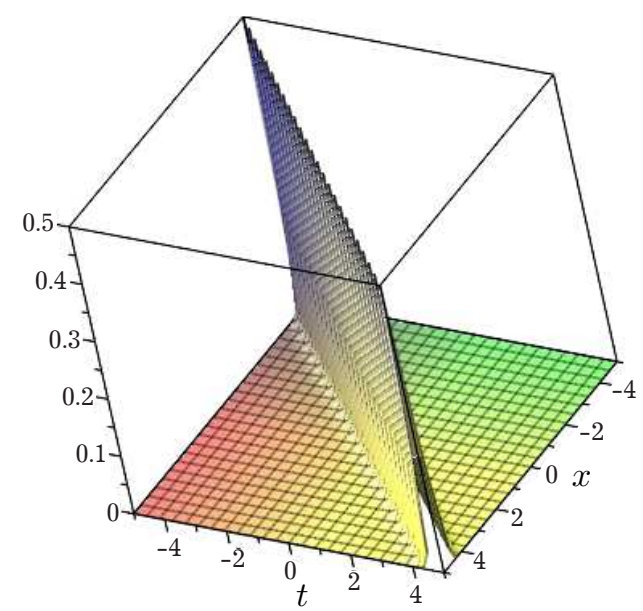

Fig. 3. Solution (23) as $a=1$ and $\varepsilon=0.1$. 


\subsection{The particular case of variable coefficients}

Let us consider a particular case, when coefficients $a_{0}(x, t), b_{0}(x, t)$ are such that $a_{0}(x, t)=a_{0}(x)$, $b_{0}(x, t)=b_{0}(x)$, where $a_{0}(x) b_{0}(x) \neq 0$ for all $x \in \mathbb{R}$. We search terms of the singular part of asymptotic one-phase soliton type solution of the singularly perturbed KdV equation (3) for $n=2$ and zero background.

The equation (17) for the function $\varphi=\varphi(t)$ becomes simple and relation (22) is written as

$$
\frac{d}{d t} A\left(\varphi, \varphi^{\prime}, t\right)=-2 A(\varphi, t)\left[2 \frac{b_{0}^{\prime}(\varphi)}{b_{0}(\varphi)}-\frac{a_{0}^{\prime}(\varphi)}{a_{0}(\varphi)}\right] \varphi^{\prime} .
$$

Equalities (17) and (24) should be compatible. It is valid for the following differential condition

$$
12 a_{0}(\varphi) b_{0}^{\prime}(\varphi)=5 b_{0}(\varphi) a_{0}^{\prime}(\varphi) .
$$

Hence, we have the relation

$$
a_{0}^{5}(x)=\rho b_{0}^{12}(x),
$$

where $\rho$ is an arbitrary real non-zero constant.

As a consequence we obtain equation for the phase function $\varphi=\varphi(t)$ in the following form

$$
\left(a_{0}(\varphi)\right)^{2 / 3} \frac{d \varphi}{d t}=\rho_{1},
$$

where $\rho_{1}$ is an arbitrary real non-zero constant.

Equation (26) has a solution in a neighborhood of the initial point $t=0$. It should be noted that a solution can exist on either finite or semi-infinite and infinite interval. It depends on the function $a_{0}(\varphi)[17]$.

As it was above mentioned, the main term of the singular part of asymptotic solution (5) is defined by the formula $V_{0}(x, t, \tau)=v_{0}(t, \tau)$, where $v_{0}(t, \tau)$ is written as $(14)$. The functions $v_{j}(t, \tau), j=\overline{1, N}$, can be found explicitly from equation (13) by the method of variations. Since $v_{j}(t, \tau) \in G_{1}^{0}$ for all $j=\overline{1, N}$, we can assume

$$
\begin{aligned}
V_{j}(x, t, \tau)=v_{j}(t, \tau)=\left(\int_{\tau_{0}}^{\tau} \Phi_{j}(t,\right. & \left.\left.\tau_{1}\right) v_{0 \tau}\left(t, \tau_{1}\right) d \tau_{1}+c_{1}\right) v_{0 \tau}(t, \tau) \int_{\tau_{0}}^{\tau} v_{0 \tau}^{-2}\left(t, \tau_{1}\right) d \tau_{1} \\
& -\left(\int_{\tau_{0}}^{\tau} \Phi_{j}\left(t, \tau_{1}\right) v_{0 \tau}\left(t, \tau_{1}\right) \int_{\tau_{0}}^{\tau_{1}} v_{0 \tau}^{-2}(t, \xi) d \xi d \tau_{1}+c_{2}\right) v_{0 \tau}(t, \tau)
\end{aligned}
$$

where the functions $\Phi_{j}(t, \tau), j=\overline{1, N}$, are defined by formula (19), $c_{1}, c_{2}$ are arbitrary real constants, $\tau_{0} \in \mathbb{R}$ is a fixed point.

\subsection{Example}

Let us show the above-mentioned algorithm of construction of the asymptotic soliton type solutions by the example. We assume the coefficients of equation (3) as

$$
a(x, t, \varepsilon)=-\left(x^{2}+1\right)^{3 / 2}, \quad b(x, t, \varepsilon)=\left(x^{2}+1\right)^{5 / 8} .
$$

Thus, we consider the equation

$$
\varepsilon^{2} u_{x x x}=-\left(x^{2}+1\right)^{3 / 2} u_{t}+\left(x^{2}+1\right)^{5 / 8} u u_{x} .
$$

Mathematical Modeling and Computing, Vol.6, No. 2, pp.374-385 (2019) 
Condition (25) holds for $\rho=-1$. In (26) we take $\rho_{1}=1$ and obtain the equation for the phase function

$$
\left(\varphi^{2}+1\right) \frac{d \varphi}{d t}=1 .
$$

For the initial condition $\varphi(0)=0$, the solution of equation (30) exists for all $t$, and it can be written as

$$
\varphi(t)=\sqrt[3]{\frac{3}{2} t+\sqrt{1+\frac{9}{4} t^{2}}}+\sqrt[3]{\frac{3}{2} t-\sqrt{1+\frac{9}{4} t^{2}}} .
$$

From formula (15) we find $A\left(\varphi, \varphi^{\prime}, t\right)=\sqrt{\varphi^{2}(t)+1}>0$.

The main term of the singular part of the asymptotic solution is written as follows

$$
V_{0}(t, \tau)=-3\left(\varphi^{2}(t)+1\right)^{-1 / 8} \cosh ^{-2} \psi(t, \tau),
$$

where $\psi(t, \tau)=\left(\varphi^{2}(t)+1\right)^{1 / 4} \tau / 2$.

Assuming $c_{0}(t)=0$ in (20), we find

$$
\Phi_{1}(t, \tau)=\frac{\varphi(t)}{\left(\varphi^{2}(t)+1\right)^{5 / 8}}\left[\frac{21}{2} \tau+\frac{45}{8} \tau \cosh ^{-2} \psi(t, \tau)-\frac{15}{4} \frac{\tanh \psi(t, \tau)}{\left(\varphi^{2}(t)+1\right)^{1 / 4}}\right] \cosh ^{-2} \psi(t, \tau) .
$$

It is easy to verify that the function $\Phi_{1}(t, \tau)$ satisfies condition (21). Moreover, $\Phi_{1}(t, \tau)$ is rapidly decreasing function of the phase variable $\tau$, i.e. $\Phi_{1}(t, \tau) \in G_{1}^{0}$.

Using formula (27), we obtain

$$
\begin{aligned}
V_{1}(x, t, \tau)=v_{1}(t, \tau)= & \frac{15}{\left(\varphi^{2}(t)+1\right)^{9 / 8}} \frac{\tau}{4}\left[\frac{37}{2}\left(\cosh ^{2} \psi(t, \tau)-\cosh ^{-2} \psi(t, \tau)\right)-1\right] \cosh ^{-4} \psi(t, \tau) \\
& +\frac{3 \varphi(t)}{\left(\varphi^{2}(t)+1\right)^{11 / 8}} \tanh \psi(t, \tau) \cosh ^{-2} \psi(t, \tau) \\
& \times\left[\frac{3}{8}+\frac{155}{8} \cosh ^{-2} \psi(t, \tau)+\frac{1}{32} \cosh ^{-4} \psi(t, \tau)+\frac{165}{2} \ln |\cosh \psi(t, \tau)|\right] \\
& -\frac{3 \varphi(t)}{\left(\varphi^{2}(t)+1\right)^{7 / 8}}\left[\frac{525}{32} \tau^{2} \cosh ^{-4} \psi(t, \tau)+\frac{5}{8}\right] \tanh \psi(t, \tau) \cosh ^{-2} \psi(t, \tau) .
\end{aligned}
$$

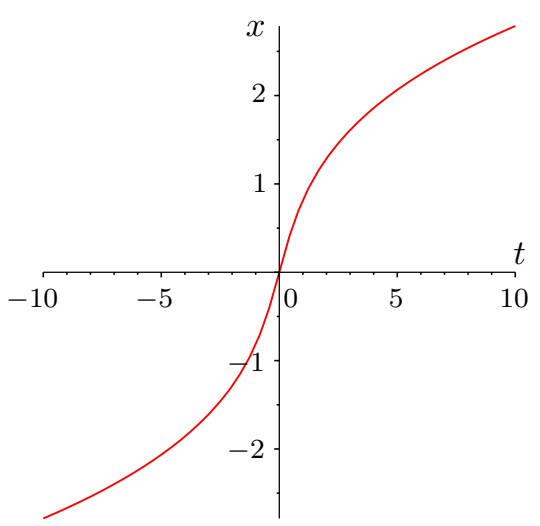

Fig. 4. The discontinuity curve (31).

We observe that $V_{1}(x, t, \tau)$ is rapidly decreasing function of the phase variable $\tau$, i.e. $V_{1}(x, t, \tau) \in G_{1}^{0}$.

In similar way we can find other terms of the singular part of the asymptotics.

Below we show figures representing the asymptotic approximation of soliton type solutions of the KdV equation (29). From their analysis one can conclude that for an adequate description of qualitative properties of the soliton type solutions of the singularly perturbed $\mathrm{KdV}$ equation with variable coefficients it is necessary to construct at least the first asymptotic approximation, i.e., the expansion containing both the main and the first terms. The latter property is compatible with asymptotic accuracy of the constructed approximate solutions [12]. 


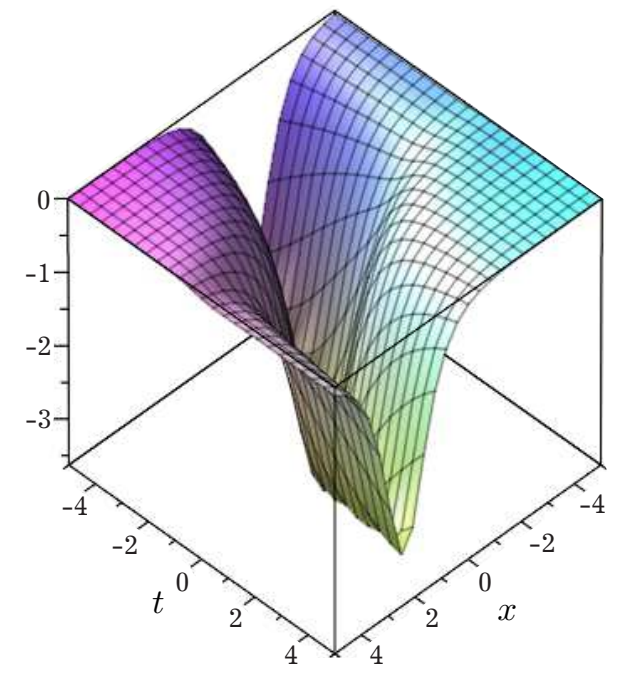

Fig. 5. The main term (32) as $\varepsilon=1$.

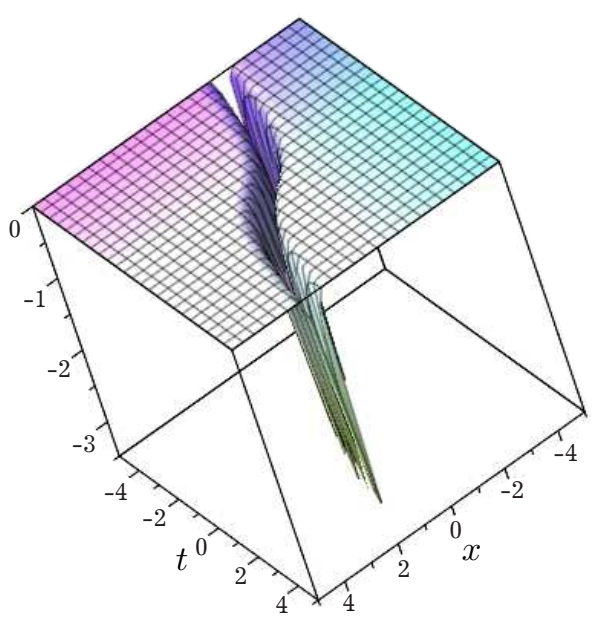

Fig. 7. The main term (32) as $\varepsilon=0.1$.

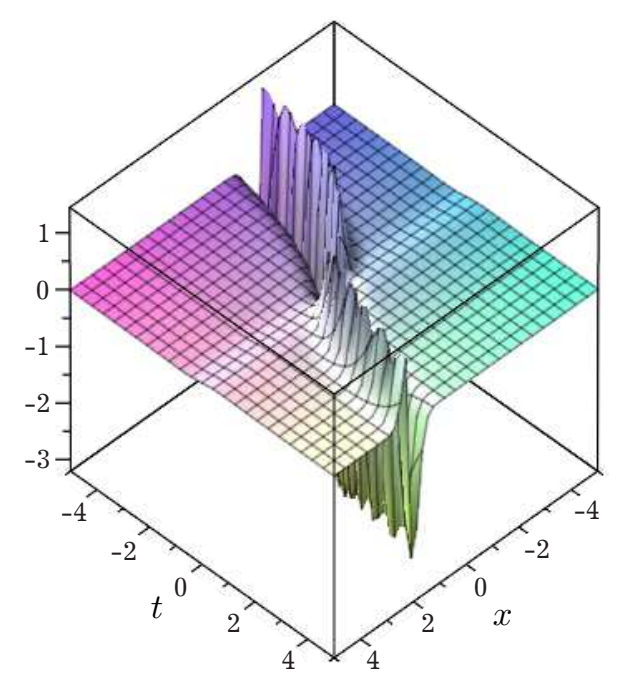

Fig. 9. The first order approximation as $\varepsilon=0.5$.

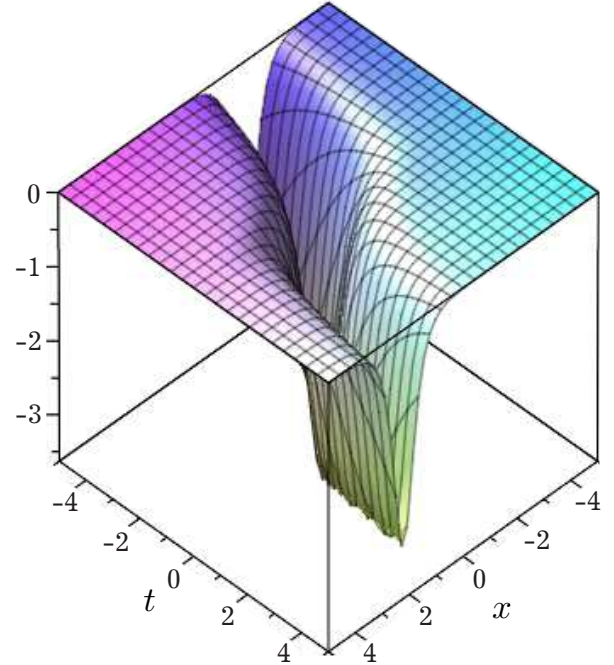

Fig. 6. The main term (32) as $\varepsilon=0.5$.

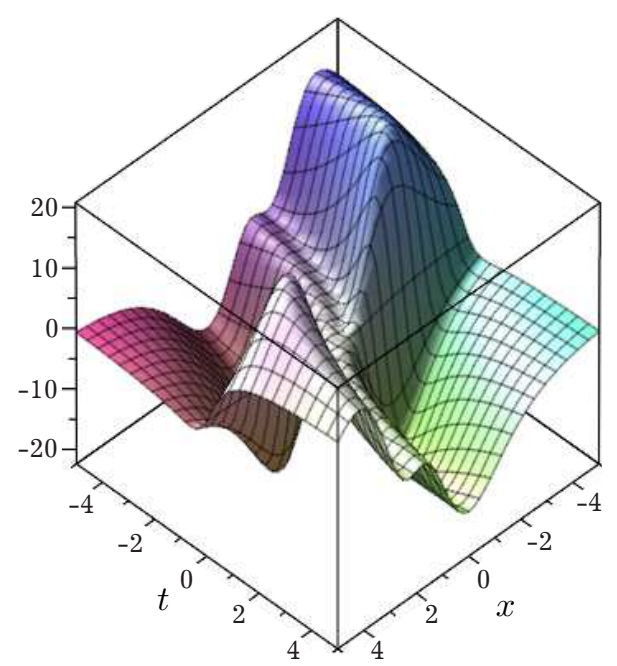

Fig. 8. The first order approximation as $\varepsilon=1$.

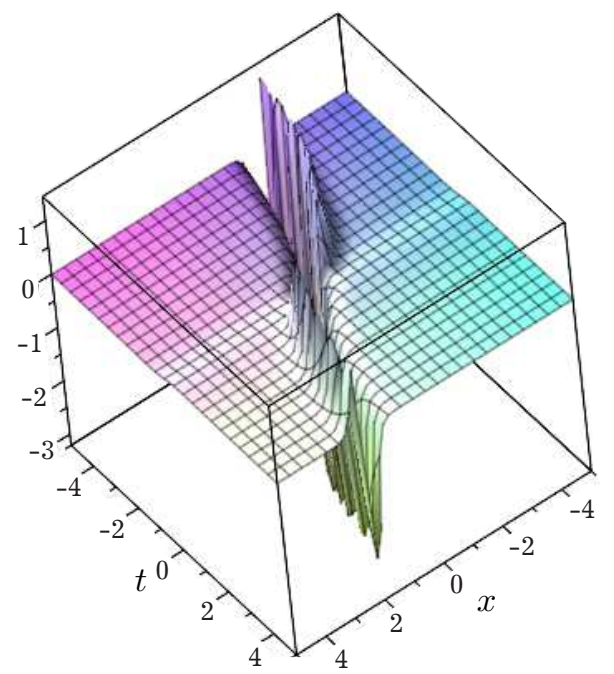

Fig. 10. The first order approximation as $\varepsilon=0.1$. 


\section{Conclusions}

The general algorithm of construction of the asymptotic soliton type solutions of the Korteweg-de Vries equation describing the wave processes in inhomogeneous media with variable characteristics and a small dispersion is given. In particular, the procedure of defining the regular and singular parts of the asymptotics is described. We obtained the conditions for coefficients of the above mentioned equation, under which the equation has asymptotic soliton type solutions.

The effect of a small parameter on the form of the constructed asymptotic solution is studied. The obtained results are shown by the example of explicit determination of the first order approximation of its asymptotic soliton type solution. In addition we consider the properties of the approximate solutions depending on a small parameter magnitude. We show that for an adequate description of qualitative properties of asymptotic soliton type solutions of the singularly perturbed KdV equation with variable coefficients it is necessary to construct at least the first asymptotic approximation, i.e., expansion containing both the main and the first terms. The latter property is compatible with asymptotic accuracy of the constructed approximate solutions.

[1] Korteweg D. J., de Vries G. On the change in form of long waves advancing in a rectangular canal and a new type of long stationary waves. Philos. Mag. 39, 422-443 (1895).

[2] Russell J. Scott. Report on waves. Reports Fourteenth Meeting of the British Association. 311-390 (1844).

[3] Zabusky N. J., Kruskal M. D. Interaction of solitons in a collisionless plasma and recurrence of initial states. Phys. Rev. Lett. 15 (6), 240-243 (1965).

[4] Kadomtsev B. B. Cooperative effects in plasmas. In Book: Reviews of plasma physics. Kluwer Academic, Springer. 22, 1-226 (2001).

[5] Toda M. Theory of nonlinear lattices. Solid State Sciences. Vol. 20. New York, Springer-Verlag (1981).

[6] Davydov A. S. Solitons in biology. In book: Modern Problems in Condensed Matter Sciences. 17, 1-51 (1986).

[7] Brizhik L., Eremko A., Piette B., Zakrzewski W. Solitons in $\alpha$-helical proteins. Phys. Rev. E. 70 (3), 031914 (2004).

[8] Newell M. C. Solitons in mathematics and physics. SIAM (1985).

[9] Maslov V.P., Omel'yanov G. A. Asymptotic soliton-form solutions of equations with small dispersion. Russian Mathematical Surveys. 36 (3), 73-149 (1981).

[10] Maslov V.P., Omel'yanov G. A. Geometric asymptotics for PDE. I. American Math. Society (2001).

[11] Miura R. M., Kruskal M. Application of non-linear WKB-method to the KdV equation. SIAM J. Appl. Math. 26 (2), 376-395 (1974).

[12] Samoilenko V.Hr., Samoilenko Yu. I. Asymptotic expansions for one-phase soliton-type solutions of the Korteweg-de Vries equation with variable coefficients. Ukr. Math. Journ. 57 (1), 132-148 (2005).

[13] Samoilenko V. Hr., Samoilenko Yu. I. Asymptotic two-phase soliton-like solutions of the singularly perturbed Korteweg-de Vries equation with variable coefficients. Ukr. Math. Journ. 60 (3), 449-461 (2008).

[14] Samoilenko V. Hr., Samoilenko Yu. I. Asymptotic $m$-phase soliton-type solutions of a singularly perturbed Korteweg-de Vries equation with variable coefficients. Ukr. Math. Journ. 64 (7), 1109-1127 (2012).

[15] Samoilenko Yu. I. Isnuvannia rozviazku zadachi Koshi dlia liniinoho rivniannia z chastynnymy pokhidnymy pershoho poriadku zi zminnymy koefitsiientamy u prostori shvydko spadnykh funktsii. Bukovinian Math. Journ. 1 (1-2), 120-124 (2013), (in Ukrainian).

[16] Golovatiy Yu. D., Kyrylych V. M., Lavrenyuk S. P. Dyferentsialni rivniannia. Lviv, Ivan Franko National University of Lviv (2011), (in Ukrainian).

[17] Kaplun Yu. I., Samoilenko V. Hr. On extendability of solutions of differential equations to a singular set. Ukr. Math. Journ. 55 (3), 450-455 (2003). 


\title{
Асимптотичні розв'язки солітонного типу для сингулярно збуреного рівняння Кортевега-де Фріза зі змінними коефіцієнтами
}

\author{
Самойленко В. Г. ${ }^{1}$, Самойленко Ю. I. ${ }^{1}$, Лимарченко В. О. ${ }^{1}$, Вовк В. С. ${ }^{1}$, Зайцева К. С. ${ }^{2}$ \\ ${ }^{1}$ Киёвсъкий націоналъний університет імені Тараса Шевченка, \\ вул. Володимирсъка, 64, Київ, 01601, Україна \\ ${ }^{2}$ Київсъкий університет імені Бориса Грінченка, \\ вул. Бульварно-Кудрявсъка, 18/2, Київ, 04053, Україна
}

У статті розглянуто сингулярно збурене рівняння Кортевега-де Фріза зі змінними коефіцієнтами, яке описує хвильові процеси в різних неоднорідних середовищах зі змінними характеристиками та малою дисперсією. Розглянуто загальний алгоритм побудови асимптотичних розв'язків солітонного типу і подано такі розв'язки для цього рівняння. Проаналізовано властивості побудованого асимптотичного розв'язку залежно від малого параметра. Результати продемонстровано на прикладах. Показано, що для адекватного опису якісних властивостей асимптотичного розв'язку солітонного типу для сингулярно збуреного рівняння Кортевега-де Фріза зі змінними коефіцієнтами необхідно будувати щонайменше перше асимптотичне наближення, тобто розклад, що містить як головний, так і перший члени асимптотики.

Ключові слова: рівняння Кортевега-де Фріза, сингулярне збурення, асимптотичний розв'язок, розв'язок солітонного типу.

2000 MSC: 35Q53, 35B25, 34E20, 34E05

УдК: 517.9 